\title{
The Effects of Number-related Factors on Entry Performance
}

\author{
Huawei Tu \\ FIT Lab, University of Swansea \\ Singleton Park, Swansea, U.K. \\ tuhuawei09@gmail.com
}

\author{
Patrick Oladimeji \\ FIT Lab, University of Swansea \\ Singleton Park, Swansea, U.K. \\ P.Oladimeji@swansea.ac.uk
}

\author{
Yunqiu Li \\ FIT Lab, University of Swansea \\ Singleton Park, Swansea, U.K. \\ yunqiu.li@swansea.ac.uk
}

\author{
Harold Thimbleby \\ FIT Lab, University of Swansea \\ Singleton Park, Swansea, U.K. \\ harold@thimbleby.net
}

Chris Vincent

UCL Interaction Centre

Gower St, London. U.K.

c.vincent@ucl.ac.uk

\begin{abstract}
Number entry is ubiquitous in user interface (UI) design, and in many applications - such as finance, aviation, healthcare - here, mitigating errors is critical. This paper examines the effects of factors such as the type of number (e.g., integer or decimal), number length (i.e., short or long) and display position (i.e., near or far) on entry errors. Until now, these factors have not been explored together. Using a touch-based numeric keypad, we found that number length influenced the probability of committing errors, while the position of presentation did not. Number type impacted user-corrected errors but not uncorrected errors. Number length, number type and display position affected input timings.

The findings provide implications for the design of both number representations (e.g., decimal point appearance) and the sociotechnical systems that surround them (e.g., training practice).
\end{abstract}

Number entry; number-related factors; entry errors; user performance

\section{INTRODUCTION}

Number entry is a very common task in a broad range of domains, including healthcare, finance and many day-to-day activities. Number entry tasks can be classified into two categories: either users input numbers stored in memory, like entering passwords, or users input numbers following instructions, for example, copying telephone numbers (Nordby et al. 2002) and programming infusion pumps (Wiseman et al. 2013). This paper focuses on the latter category of input. These tasks are visually and cognitively demanding, as users need to view strings of digits then remember them in short-term memory.

Number entry tasks are composed of planning and acting, as in the GOMS model (Card et al. 1983), which has been widely applied to analyzing the complexity of interactive systems, including cognitive information processing activities. Here planning is the process of organizing action steps required for numeric entry, that is viewing numbers and remembering them in short-term memory, and acting is the process of performing these steps, that is interacting with the UI. The relation between planning and acting is a fundamental basis for all

\begin{tabular}{|c|c|c|}
\hline & Number-related Factors & Research State \\
\hline \multirow{3}{*}{ Numeric Attributes } & Length: Short or Long & $\begin{array}{l}\text { Miller, G. A. (1956); } \\
\text { Nordby, K., et al. (2002). }\end{array}$ \\
\hline & $\begin{array}{l}\text { Type: Integer or } \\
\text { Decimal }\end{array}$ & None \\
\hline & $\begin{array}{l}\text { Magnitude and } \\
\text { Frequency }\end{array}$ & $\begin{array}{l}\text { Wiseman, S., et al. } \\
\text { (2013). }\end{array}$ \\
\hline \multirow{2}{*}{$\begin{array}{l}\text { Presentation } \\
\text { Variables }\end{array}$} & $\begin{array}{l}\text { Presented Position: } \\
\text { Near or Far }\end{array}$ & None \\
\hline & Font Appearance & $\begin{array}{l}\text { Nacenta, M., et al. (2012); } \\
\text { Thimbleby, H. (2013). }\end{array}$ \\
\hline
\end{tabular}

Table 1: Number-related factors for numeric entry interaction.

interaction (Card et al. 1983; O'hara and Stephen 1999) and thus has a significant influence over numeric entry.

Previous studies (Colle and Hiszem 2004; Miller 1956; Nordby et al. 2002) show that some numeric attributes (e.g., number length) can affect the way that users view and mentally process numbers (e.g., how they group digits for short or long numbers). However, it is not clear how these factors influence the bigger picture of number entry performance at the level of planning and acting. Therefore we 
investigated the effects of factors such as number length, type and display position (see Table 1) that have not previously been explored in the literature. While the effects of number length have been explored with respect to user ability for immediate recall (Miller 1956; Nordby et al. 2002), research is also lacking on the effect of number length on the processes of planning and acting for numeric entry. Furthermore, the impact of number type and position remains unclear. Understanding these effects can help establish a solid foundation to improve both performance and design.

This paper explores two fundamental questions: first, what are the effects of these factors on entry errors and, second, how do these factors influence entry processes - planning and acting?

We conducted a study using a touch-based numeric keypad, controlling four factors: number length, number type, display position, and key size. We present the results of the experiment with a detailed discussion of the design implications for improving numeric entry interaction.

\section{RELATED WORK}

Number entry has attracted widespread interest from two main directions: UI design and numeric representation. The role of $U I$ design is recognized for improved entry performance, resulting in many studies. Several studies have explored how key size, shape and UI layout affect number entry performance (Colle and Hiszem 2004; Sears et al. 1993). In addition, others have investigated the effects of UI types (e.g., numeric keypad versus updown keypad) on speed, errors and severity of errors (Oladimeji et al. 2011, 2013). There have also been suggestions concerning alternative input techniques to support numeric entry, including handwriting and gesture input with a pie menus (Isokoski and Käki 2002; MacKenzie et al. 1994). On the other hand, regarding numeric representation, psychological studies have concentrated on examining the effects of number length and digit-grouping formats on immediate recall (Miller 1956; Nordby et al. 2002). The magnitude and frequency of numbers used in hospitals have been studied to improve number entry UI design for medical devices (Wiseman et al. 2013). Furthermore, some studies have investigated syntactic and visual aspects of numbers to improve numeric representation (Nacenta et al. 2012; Thimbleby 2013). It should also be noted that numbers are used for different purposes;for example, for a credit card PIN number, the number entered has to be exactly correct, whereas for many other values (e.g. cooking time), numbers entered

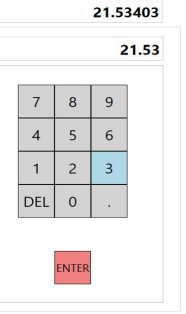

(a)

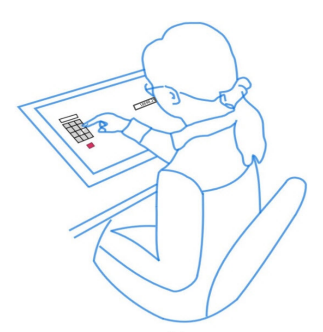

(b)
Figure 1: (a) Experiment UI. Instruction numbers were shown above the entry interface (near position). (b) A participant in the experiment environment. Instruction numbers were shown on the right of the entry interface (far position).

within a few percent of the intended value may be fine.

This varied research background provides valuable results and guidelines to improve number entry performance. Unfortunately, our literature review shows that little attention has been paid to examining number-related factors, especially number length, type and display position, on user performance. The present study aims to fill this gap by exploring the effects of these factors.

\section{EXPERIMENT}

\subsection{Interface, Materials and Apparatus}

The UI was a numeric keypad (see Figure 1a). Twelve square keys in gray were arranged without key spacing. Three key sizes $(5.4,12.15$ and $18.9 \mathrm{~mm}$ per side represent small, medium and large sizes respectively) were used in the experiment (Colle and Hiszem 2004), as we aimed to examine number-related factors with different key sizes. For small, medium and large key sizes, the text font size of the key was $2.7,4.05,5.4 \mathrm{~mm}$ respectively.

A red button (measuring $18.9 \mathrm{~mm}$ per side) was placed below the number pad with a constant gap of $18.9 \mathrm{~mm}$, for controlling the start and end of a trial. The text of the control button was set as "START" initially. Pressing the start button indicated starting a trial. Once a trial started, the button text changed to "ENTER." Participants were asked to click this button when they finished a trial. The button text then changed to "START" again, prompting participants to start the next trial.

The touchscreen used the lift-off button activation mechanism with visual feedback. The entry interface was to the left side of the screen (see Figure 1b), and the instruction (font size $6.75 \mathrm{~mm}$ ) was shown on the right side of the entry interface at a distance of $378 \mathrm{~mm}$ (far position) or above it at a distance of $8.1 \mathrm{~mm}$ (near position). For the far position, a 
pilot study was conducted to determine the distance between the entry interface and the instruction number to ensure that participants needed to switch their visual attention across the two separate physical spaces.

Forty-eight randomly generated numbers were used in the experiment. All numbers were different. 24 numbers were integer type (with no decimal point) and the others were decimal type (that is, numbers with digits before and after a decimal point). Numbers fell into two further categories according to their lengths: for both integer and decimal numbers, there were twelve short numbers (six 2-digit numbers and six 3-digit numbers) and twelve long numbers (six 6-digit numbers and six 7digit numbers). For decimal numbers, the location of the decimal point was evenly distributed amongst the digits. The number length was determined based on previous study by Nordby et al. (Nordby et al. 2002) which showed that immediate recall performance degraded rapidly beyond 6 digits. We accounted for effects of the spatial position of numbers across the different conditions. We did this by creating numbers that were balanced in terms of the position of the digits in the keypad.

The experiment was conducted on a Dell S2340T multi-touch monitor connected to an Asus S550c laptop. The monitor was $53.32 \times 31.20 \mathrm{~cm}$ with a resolution of $1,920 \times 1,080$ pixels. The response time of the touch screen was $8 \mathrm{~ms}$. During the experiment, the touch screen was mounted on a fixed surface tilted at $30^{\circ}$ to the surface of the desk to reduce fatigue (Sears et al. 1993).

\subsection{Participants}

Sixteen participants (5 male) from 24-47 years of age $(\mu=33.6, \sigma=7.4)$, were recruited for the study. Three were left-handed. All had prior experience using touch screens and were familiar with number entry on numeric keypads. All of them had normal or corrected to normal vision and no motor impairments. All participants were numerate university graduates or staff.

\subsection{Experiment Design}

The experiment was a within-subject design. There were four independent variables: position of instruction numbers (near and far), number length (short and long) and number type (integer and decimal), key size (small, medium, large). The dependent variables were corrected errors, uncorrected errors and entry time.

\subsection{Procedure}

The experiment consisted of a training phase and an experimental phase. In the training phase, participants were instructed how to perform the task, they were then asked to enter 6 numbers for each combination of the three key sizes and the two positions. During the experiment, participants sat in a chair in front of the experiment device (see Figure 1b). In each trial, an instruction number was shown either on the right of the entry interface or above it. Participants were asked to use the index finger of the dominant hand to enter the presented number as quickly and accurately as possible. After entering the number, participants were instructed to press the ENTER button to confirm the current trial and then press the START button for the next trial. Participants were encouraged to correct entry errors if they detected any. Each participant completed 6 blocks consisting each of the 48 instruction numbers for each combination of the three key sizes and the two positions. The order of the combination of button sizes and positions was counterbalanced across the participants. The order in which the numbers were presented for each participant was randomized. In summary, the experiment consisted of 16 participants $\times 3$ key sizes $\times 2$ positions $\times 48$ numbers $=4,608$ trials.

\subsection{Results and analysis: Errors}

Entry errors in the experiment were classified into uncorrected errors and corrected errors (Oladimeji et al. 2011). Uncorrected errors were committed if the input number was different from the instruction number, and this error was not caught before the ENTER button was pressed. Note the definition of uncorrected errors differs from ones for text entry (Wobbrock and Myers 2006). We used this definition of uncorrected errors to conform to cases in safety fields, where an error is committed no matter the number of wrong digits (Thimbleby and Cairns 2010). Corrected errors were committed if the DEL key had been used during the entry sequence, but the input number was the same as the instruction number when the ENTER button was pressed. The data of error rates were not normally distributed, so they were analyzed using the Wilcoxon signed-rank test for two pairs and Friedman test for three pairs. Post hoc analysis with Wilcoxon signed-rank tests was conducted with the Bonferroni correction (the adjusted significance level was $0.05 / 3=0.017$ ).

The uncorrected error rate was calculated by dividing the sum of uncorrected errors by the number of all trials. In total, there were 61 instances of this error. No significant effect was found for position $(Z=$ $-0.27, p=0.79)$, number type $(Z=-0.04, p=0.97)$, or key size $\left(\chi^{2}(2)=3.33, p=0.19\right)$. However, it was found that long numbers $(\mu=0.02)$ correlated with significantly more errors than short numbers $(\mu=0.01, Z=-2.40, p<0.05)$. 


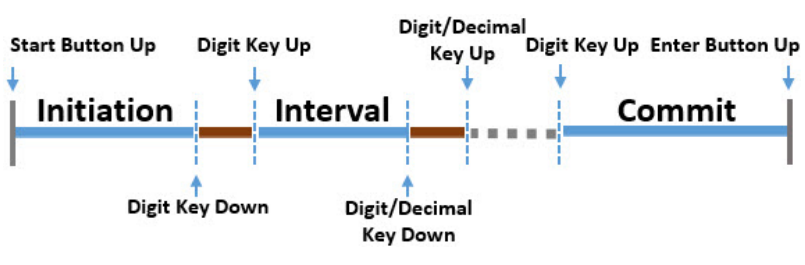

Figure 2: Keystroke-level elements of numeric entry process.

The corrected error rate was calculated as the ratio of the sum of corrected errors to the number of all trials. The number of this error was 294. There were a significant effect for number length $(Z=$ $-3.52, p<0.01)$, with long numbers $(\mu=0.31)$ having more errors than short numbers $(\mu=0.09)$. In addition, a significant effect was revealed for key size $\left(\chi^{2}(2)=26.00, p<0.01\right)$. Post hoc analysis revealed a significant difference between all three key sizes $(p<0.017)$ except between medium and large ones $(p=0.02)$. The mean rate was 0.38 , 0.13 and 0.07 for small, medium and large sizes respectively. Interestingly, for position, no significant effect was found $(Z=-0.63, p=0.53)$.

For number type, one may argue that different character lengths of decimal numbers and integer numbers might affect statistical effects on corrected error analysis. To eliminate such effects, we normalized corrected errors by calculating the ratio of the number of corrected errors to the length of the number string in a trial. A normalized corrected error rate was then calculated by dividing the sum of normalized corrected errors by the number of all trials. Decimal type $(\mu=0.04)$ resulted in significantly higher error rate than integer type $(\mu=0.03, Z=$ $-2.17, p<0.05)$.

\subsection{Results and analysis: Time}

For trials with errors, additional correction presses penalised time performance. These trials cannot provide valid data for time analysis in terms of keystroke level elements (i.e., initiation, interval, and commit phases). Therefore, experimental trials with uncorrected or corrected errors (5.1\% of all trials) were removed from the experimental data for time analysis. Repeated measures ANOVA and post hoc comparisons with the Bonferroni adjustment were used in the analysis presented below. We aimed to examine the effects of the number-related factors on keystroke-level elements of entry process (see Figure 2).

Initiation time was defined as the duration from the release of the START button to contact with the first digit key. A significant main effect was found on initiation time for number type $\left(F_{1,15}=7.30, p<\right.$ $0.05)$, number length $\left(F_{1,15}=43.10, p<0.01\right)$, position $\left(F_{1,15}=213.96, p<0.01\right)$, and key size $\left(F_{2,30}=29.63, p<0.01\right)$. The mean initiation time was $1,003 \mathrm{~ms}$ and $1,024 \mathrm{~ms}$ for integer and decimal types, $916 \mathrm{~ms}$ and $1,111 \mathrm{~ms}$ for short and long numbers, and $903 \mathrm{~ms}$ and 1,124 ms for near and far positions. For key size, the mean value was $1,120 \mathrm{~ms}, 963 \mathrm{~ms}$ and $957 \mathrm{~ms}$ for small, medium and large sizes. Post hoc comparisons reveal a significant difference between each two sizes $(p<$ $0.01)$ except medium and large sizes $(p=1.00)$.

Interval time was defined as the duration from the release of the current digit or decimal keys to the contact of the next digit or decimal keys. The interval time was comprised of finger movement time, and the time cost of choice and motor programming process. For each entry trial the average interval time $\left(\mathrm{Al}_{\text {Time }}\right)$ was calculated as the ratio of the sum of every interval time to the length of the number string (including the decimal point if any) minus one. There was a significant effect for number type $\left(F_{1,15}\right.$ $=4.57, p<0.05)$, number length $\left(F_{1,15}=92.39\right.$, $p<0.01)$ and position $\left(F_{1,15}=10.50, p<0.01\right)$. Small, medium and large key sizes had a mean $\mathrm{Al}_{\text {Time }}=472 \mathrm{~ms}, 294 \mathrm{~ms}$ and $290 \mathrm{~ms}$ respectively. A significant difference was found between each two sizes $(p<0.01)$ except medium and large sizes $(p=1.00)$.

Commit time was calculated as the duration from the release of the last digit key to the release of the ENTER button. There was a significant main effect on commit time for number length $\left(F_{1,15}=43.63, p<\right.$ 0.01) (524 ms for shot numbers and $662 \mathrm{~ms}$ for long numbers). A significant main effect was also found for key size $\left(F_{2,30}=13.65, p<0.01\right)$. The mean time was $645 \mathrm{~ms}, 555 \mathrm{~ms}$ and $579 \mathrm{~ms}$ for small, medium and large key sizes. There was a significant difference between each two sizes $(p<0.01)$ except medium and large ones $(p=0.24)$.

\section{DISCUSSION}

\subsection{Number-related Factors and Entry Errors}

Reduction of error is important in many safety critical tasks. Unnoticed errors by definition cannot be managed by users and may consequently lead to adverse events (Thimbleby and Cairns 2010). Furthermore, it is difficult to build a predictive model (like ones for text entry) that suggests corrections for numeric entry errors because numeric errors are more ambiguous than those in text entry. As we anticipated, corrected errors increase as the number length increases; this phenomenon is predicted by projecting the probability of error in a single pointing task to the probability of error in a single task involving $n$ pointing subtasks. Nevertheless it is interesting that long numbers elicit more unnoticed 
errors than short numbers. While the reason for this remains to be explored, it suggests that the probability of error detection is inversely proportional to the length of an input sequence. The analysis emphasizes the effectiveness of using chunking for error reduction, for instance presenting 20130517 as 20,130, 517 or 20130517 as in (Miller 1956), and shows a need to understand effects of number length on committing unnoticed errors. This will be a useful topic for further research.

Decimal type numbers had more corrected errors than integer type numbers, which prompted further investigation of the trials containing corrected and uncorrected errors. We found that for decimal type, $27.6 \%$ of trials had corrected errors related to missing the decimal point, and $30 \%$ of trials had uncorrected errors associated with missing the decimal point. This could be because entry tasks are visually and cognitively demanding; the decimal point may effect the way participants viewed and mentally processed numbers. Regarding the aspect of mental process, the decimal point may influence how users group digits: some participants reported that they tended to select the decimal point as a delimiter for grouping digits. These effects can be reflected by the analysis of average interval time: decimal type had longer time than integer type. On the other hand, compared to digits, the decimal point is usually smaller in numbers, which may produce a higher probability of missing it (especially for dataentry tasks). Based on the results, we propose that decimal points should be made to be prominent in numbers (e.g., 20 • 5). Additionally, it is worth exploring designs that do not require users to enter the decimal point, or which scaling numbers so that they are integers. Other possibilities are to avoid conventional number entry altogether, such as using 5-key Uls (Oladimeji et al. 2013). This begs experiments comparing the various schemes, a topic for future research.

The results provide compelling evidence that while the position of the instruction numbers influenced time performance, it did not have a significant impact on errors. This enhances our understanding of number entry errors and also implies guidelines for research work and real practice. Taking programming infusion pumps on hospital wards as an example, nurses tend to do the programming while viewing prescription forms, which may be placed in far or near positions depending on the context. From our results, it is reasonable to expect that they would achieve a similar entry accuracy. This information may be useful when it comes to training users (e.g., nurses), and forms the stimulus for future study (e.g., why did the two positions not impact on error rates).

\subsection{Planning and Acting}

In GOMS, sequences of entry actions are organised by a mental planning process (Card et al. 1983). Unlike text entry, where most if not all words entered are stored in long-term memory (Wobbrock and Myers 2006), number transcription requires users to view instruction numbers and remember them in short-term memory. Hence, users adopt different strategies for processing numbers and text, such as grouping digits for long numbers (Nordby et al. 2002) but use different mechanisms for words.

Number length, type and position influenced the number entry time, which allowed us to infer the process used to plan and act when entering numbers. The longer duration between key strokes for long numbers, decimal type and far position were caused by the requirement of more extensive planning or programming of the motor sequences (e.g., chunking) as well as visual search and movement (Colle and Hiszem 2004). In addition, we found that the planning time in the initiation step and the checking time in the post-completion step correlated significantly with the number length. The longer the number is, the more time the participant took in planning and checking phases. This behavior provides insight into how users deal with number entry tasks at the levels of planning and checking and also emphasizes the importance of planning and checking in number entry process. For work-intensive environments, where users may not dedicate enough time into planning and checking numeric entry, we suggest that number entry interface design should consider providing features to encourage users to spend more time and pay more attention in the initiation and commit phases, such as the lockout time strategy proposed in (O'hara and Stephen 1999).

\subsection{Number Entry Interface Design}

The effect of key size on number entry performance has generated considerable research interest (Colle and Hiszem 2004). This study investigated three key sizes in terms of 3 time-related measures and 2 error-related measures. These measures reflect entry errors as well as planning and executing processes during numeric entry in terms of keystroke-level elements. Keys with a side length of 12.15 and $18.9 \mathrm{~mm}$ had similar performance, indicating $12.15 \mathrm{~mm}$ is sufficiently large for lift-off key entry. The result and methodology presented here could be used more widely for number entry interface design. 


\section{CONCLUSIONS}

We examined the effects of number-related factors (number length, number type and position) and key size on number entry performance. In terms of insights for $\mathrm{HCl}$ and $\mathrm{UI}$ design, our results suggest that user interfaces for number entry should: make the decimal point larger; see if the task can be changed to eliminate decimal points; provide a DEL key; provide features to encourage users to pay more attention in the initiation and commit phases. We found that number length increased errors. In areas that are safety critical Uls should be designed to reduce number length if at all possible. For example, in healthcare, the use of off-the-shelf calculators encourages nurses to use 8 digit numbers, when only 2 or 3 digits would be sufficient for most clinical purposes (Wiseman et al. 2013).

More specifically, we found that number length influenced the probability of committing errors (i.e., long numbers led to more uncorrected and corrected errors than short numbers), while position of presentation did not. Number type and key size did not affect uncorrected error rates but did affect corrected errors. Number length, number type, position and key size affected the initiation phase and inter-key-press durations during number entry: long numbers, decimal type, far position and large sizes resulted in longer initiation and interval time than short numbers, integer type, near position, and small sizes respectively. For the post-completion phase, long numbers and large key sizes had longer commit times than short numbers and small sizes respectively. In future work, we would like to examine number-related factors with a physical interface and consider individual differences in experiment design.

Our findings offer an understanding of the relation between number-related factors and numeric entry, and also provide insight into future work for improving number entry performance.

\section{ACKNOWLEDGMENTS}

Funded as part of CHI+MED: Multidisciplinary Computer-Human Interaction research for design and safe use of interactive medical devices project EPSRC Grant Number EP/G059063/1.

\section{REFERENCES}

Card, S., T. Moran, and A. Newell (1983). The psychology of human-computer interaction. L. Erlbaum Associates.

Colle, H. and K. Hiszem (2004). Standing at a kiosk: Effects of key size and spacing on touch screen numeric keypad performance and user preference. Ergonomics 47(13), 1406-1423.

Isokoski, P. and M. Käki (2002). Comparison of two touchpad-based methods for numeric entry. In
Proceedings of the ACM Conference on Human Factors in Computing Systems, Minneapolis, CHI '02, pp. 2532. ACM

MacKenzie, I. S., B. Nonnecke, S. Riddersma, C. McQueen, and M. Meltz (1994). Alphanumeric entry on pen-based computers. Int. J. Hum.-Comput. Stud. 41(5), 775-792.

Miller, G. A. (1956). The magical number seven plus or minus two: Some limits on our capacity for processing information. Psychological review 63(2), 81-97.

Nacenta, M., U. Hinrichs, and S. Carpendale (2012). Fatfonts: Combining the symbolic and visual aspects of numbers. In Proceedings of the International Working Conference on Advanced Visual Interfaces, AVI '12, New York, NY, USA, pp. 407-414. ACM.

Nordby, K., R. K. Raanaas, and S. Magnussen (2002). The expanding telephone number part 1: Keying briefly presented multiple-digit numbers. Behaviour \& Information Technology 21(1), 27-38.

O'hara, K. P. and J. P. Stephen (1999). Planning and the user interface: The effects of lockout time and error recovery cost. International Journal of HumanComputer Studies 50, 41-59.

Oladimeji, P., H. Thimbleby, and A. Cox (2011). Number entry interfaces and their effects on error detection. In Human-Computer Interaction - INTERACT 2011 , Volume 6949 of Lecture Notes in Computer Science, pp. 178-185. Springer Berlin Heidelberg.

Oladimeji, P., H. Thimbleby, and A. Cox (2013). A performance review of number entry interfaces. In Human-Computer Interaction - INTERACT 2013, Volume 8117 of Lecture Notes in Computer Science, pp. 365-382. Springer Berlin Heidelberg.

Sears, A., D. Revis, J. Swatski, R. Crittenden, and B. Shneiderman (1993). Investigating touchscreen typing: The effect of keyboard size on typing speed. Behaviour \& Information Technology 12, 17-22.

Thimbleby, H. (2013). Reasons to question seven segment displays. In Proceedings of the SIGCHI Conference on Human Factors in Computing Systems, CHI '13, New York, NY, USA, pp. 1431-1440. ACM.

Thimbleby, H. and P. Cairns (2010). Reducing number entry errors: Solving a widespread, serious problem. Journal of The Royal Society Interface 7(51), 14291439.

Wiseman, S., A. L. Cox, and D. P. Brumby (2013). Designing devices with the task in mind: Which numbers are really used in hospitals? Human Factors: The Journal of the Human Factors and Ergonomics Society 55(1), 61-74.

Wobbrock, J. O. and B. A. Myers (2006). Analyzing the input stream for character-level errors in unconstrained text entry evaluations. ACM Trans. Comput.-Hum. Interact. 13(4), 458-489. 